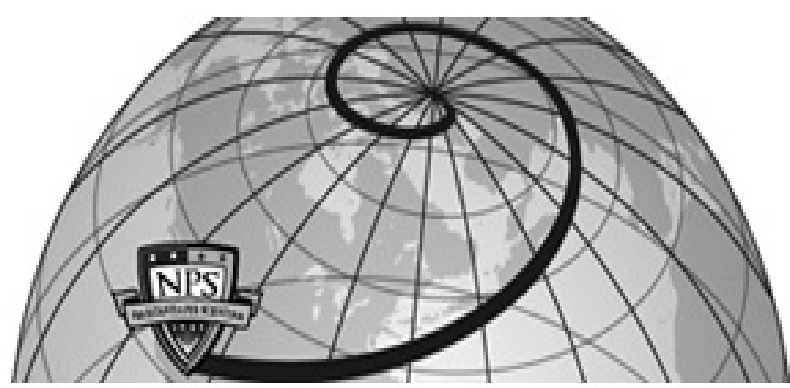

Calhoun: The NPS Institutional Archive DSpace Repository

\title{
Options-Based Multi-Objective Evaluation of Product Platforms
}

Gonzalez-Zugasti, Javier P.; Otto, Kevin N.; Whitcomb, Clifford A.

https://hdl.handle.net/10945/43453

This publication is a work of the U.S. Government as defined in Title 17, United States Code, Section 101. Copyright protection is not available for this work in the United States.

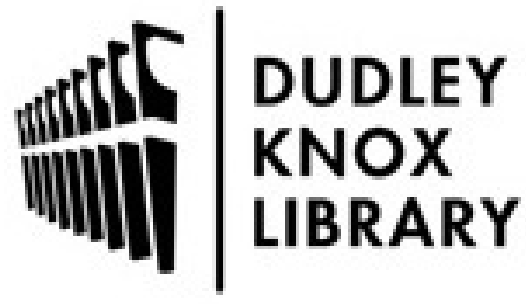

http://www.nps.edu/library
Calhoun is the Naval Postgraduate School's public access digital repository for research materials and institutional publications created by the NPS community. Calhoun is named for Professor of Mathematics Guy K. Calhoun, NPS's first appointed -- and published -- scholarly author.

Dudley Knox Library / Naval Postgraduate School 411 Dyer Road / 1 University Circle Monterey, California USA 93943 


\title{
Options-Based Multi-Objective Evaluation of Product Platforms
}

Javier P. Gonzalez-Zugasti, Kevin N. Otto, and Clifford A. Whitcomb

\begin{abstract}
Hbstract
A platform is the set of elements and interfaces that are common to a family of products. Design teams must choose among feasible platform concepts upon which a product family could be based, often involving new technologies. Multiple performance objectives need to be considered. A standard approach is to convert the performance outcomes into financial figures, which can then be weighed against the required investments. However, it is not always possible to transform performance outcomes (benefits) into monetary terms, such as in defense or highly technical projects. A multi-objective form of real-options-based platform selection is developed. Systems are compared based on multiple technical and economic goals, incorporating uncertainty by representing the unknown factors during the subsequent development process with probability distributions. The range of uncertain outcomes is integrated into single expected measures of effectiveness, which can then be applied to select the most appropriate platform and set of support product variants. An application to the design of platform-based families of naval high-speed ships is shown.
\end{abstract}

\section{Introduction}

Increasingly, organizations are faced with a need to offer a higher variety, more customer-focused products, all the while facing increasing competition, limited resources, and higher complexity of designs. In order to be able to produce a higher number of offerings, many have turned to basing a product portfolio on a common set of elements and a common structure, what is generally called a platform architecture. Evaluating what makes an effective platform from many alternative structures is considered. In this paper, a multi-objective form of real-options evaluation for platforms that support multiple products, incorporating uncertainty, is created and described.

A product platform is the set of common elements, interfaces, and processes that are shared by the different offerings within a product family, or the variants (Meyer and Lehnerd 1997). Using a platform approach to designing multiple products has several advantages. First and most apparent is the cost savings that can be achieved by sharing parts and manufacturing processes. Automotive companies report saving billions per year on single platform-based families (Bremner 1999). Second, development costs are drastically reduced. Ford reports dramatic cost savings from switching between designing V-8 engines in a one-at-a-time fashion to a platform-based strategy (Sanderson 1991). Third, there are also decreased risks that come from using product platforms, because the shared core of the products can be refined over successive offerings. Fourth, and possibly most important, is the advantage platforms provide as far as providing more variety. Sony outpaced all its competitors in the number of Walkman ${ }^{\mathrm{TM}}$ products it offered by basing them on three core platforms (Sanderson and Uzumeri 1995). A more 
complete coverage of the pros and cons of using platforms can be found in Meyer and Lehnerd (1997), along with several other examples.

The earliest and one of the most challenging questions when designing a family of products based on a platform is what to include in the platform itself. Many platforms can be envisioned from which a product family could be derived, each alternative platform having a different choice of what is shared and what is individually designed for each product. Several methods can be used to create these platform alternatives and evaluate their technical effectiveness, one of which will be later reviewed and applied in the Approach section. However, even when alternatives are all equally good from a technical standpoint, there are many unknowns during the development of the product family that can make one of those choices more preferable. For example, a platform with slightly higher costs but also higher flexibility to become a basis for more future variants may be preferred over a less costly platform design that is less flexible to uncertainties. Making such a selection requires a model of the development of the product family that incorporates the uncertainties present. It is also represents the decision that system architects must make when configuring a platform.

One way to select from among competing designs is to measure the outcomes of creating each product family. If these outcomes or benefits can be quantified in monetary terms, there exist many techniques to make an evaluation of the value of each design to the organization, such as net present value (NPV) calculations, or realoptions models.

For many applications, however, quantifying outcomes in terms of monetary value is impossibly difficult. For example, a company may wish to take into consideration not just quantifiable short-term profitability but also long-term profitability based on investment in new technologies whose monetary value cannot be reliably assessed. As another example, the added monetary value to the military defense of reduced casualties, reduced mission times, or more heavily defeated adversaries also cannot be easily quantified. Instead, all that can be determined with confidence is the expected military effectiveness on various criteria such as speed, coverage, and technical engineering criteria of the considered system. Such systems with nonmonetary benefits are addressed in this paper.

Quantifying the outcomes of development projects when multiple performance criteria are involved is more difficult than formulations that consider monetary value, because the multiple categories must be weighed against one another in order to combine them into a single performance metric, and these weightings are subjective. The problem is particularly acute for non-marketed products because there are no market data to quantify the value of offerings with different attributes. In this paper, a multiobjective form of real-options-based evaluation is developed, which is useful for considering platforms that support multiple technical product variants. The work is demonstrated using a set of notional advanced hull forms, where the variants define possibilities for multiple surface ship applications.

\section{RELATED WORK}

In the product design literature, there are several design and manufacturing strategies for offering variety that begin with commonality metrics that fundamentally address product complexity issues. Martin and Ishii (1997) present ideas on delaying when to introduce variety in a production line. On the demand side, Yu, GonzalezZugasti, and Otto (1999) map customer demand variety to effective portfolio architecture, which was used by Polaroid and Xerox Corporation.

There are also several model-based approaches to designing different kinds of product platforms. Krishnan, Singh, and Tirupati (1998), Simpson, Maier, and Mistree (1999), Mistree, Hughes, and Bras (1993), and Allen, Mistree, and $\mathrm{Al}$ (1992), developed optimization models to design families of products that have identical architectures but scale in size. Krishnan et al.'s 
work was used by Compaq Corporation in the design of notebook computers.

Other works consider functional approaches to platform analysis, partitioning. and aggregating functions into modules. Stone, Wood, and Crawford (1998). developed rules to partition a single product into possible modules. Zamirowski and Otto (1999) then developed further rules to partition an entire product family, and applied the work at Xerox. Dahmus and Otto (2001) then developed the modularity matrix to help represent alternatives generated, which has been used at ITT Corporation among others.

Most related to this paper are design optimization approaches that have been developed to consider the more general case of modular platforms. Gonzalez-Zugasti, Otto, and Baker (1998) and Nelson, Parkinson, and Papalambros (1999) developed formulations to design a platform with a family of variants. Fujita, Sakaguchi, and Akagi (1999) consider optimizing the selection of fixed-design modules from catalogs of existing modules. Gonzalez-Zugasti and Otto (2000) combine these three works to form a general optimization of the number and configuration of possible interchangeable modules that form a family. Gonzalez-Zugasti's work was used at NASA JPL to evaluate architectures for deep space probes.

Real-options concepts are applied to model outcomes and uncertainty of a design/development process. Similar techniques have been reported in the management literature for projects with outcomes that can be transformed into monetary units. For example, Faulkner (1996) calls this general approach "Options Thinking," and applies it to the development of imaging products. Neely (1998) suggests a similar approach and applies it to automotive R\&D projects. Several other researchers have shown that a real-options approach to quantifying the outcomes of a development project can yield a different and more accurate funding decision than simpler, more traditional metrics such as discounted cash flow (DCF) or net present value (NPV) calculations
(Trigeorgis and Mason; Brealey and Myers 1991; Dixit and Pindyck 1994). This difference stems from the ways the methods account for uncertainty during the development project. A simple DCF analysis assumes that all the uncertainty is fixed at the present values, so all the funding decisions for a project can be determined at the present time.

\section{PAPER OUTLINE}

The subsequent section will discuss the theoretical background for an evaluation and selection method for platform designs. The Approach section will then describe a method for creating technically feasible platform alternatives, and then evaluate them on multiple criteria under uncertainty to select the most valuable. The Application section will show an example of applying the proposed method to the selection of a platform design for a new Navy high-speed ship.

\section{Platform Development}

Many possible designs could be found to create a product family based on a common platform; however, the one that yields the best combination of multiple objectives, both in technical and economic performance, is the one desired. This can be expressed in general terms as trying to maximize the value of the development project, which can then be expanded into multiple objectives.

\section{UNDERLYING OBJECTIVE}

If a mapping of multiple attributes into monetary terms is constructed, then the value of a platform concept to the firm is simply the sum of the benefits realized from the derived set of products minus the investments (or costs) to develop and market (or operate) them. This would include all lifecycle factors that wish to be considered (e.g., design, manufacturing, maintenance, disposal, etc.).

To develop this formulation, value is derived first for a single product and is subsequently expanded to the formulation for a set of products in the platform. Defining $\vec{x}_{i}$ as the vector of design variables for a product $i$, the general 
objective of maximizing value can be expressed as finding:

$$
\begin{aligned}
\vec{x}_{i}^{*} & : V\left(\vec{x}^{*}\right)=\max _{\vec{x}_{i}} \text { Value } \\
& =\sum \text { Benefits }-\sum \text { Investments }
\end{aligned}
$$

subject to meeting a set of constraints, $\vec{g}$, that ensure that each product meets its targets and is within its limits, $\vec{\tau}$ :

$$
\vec{g}\left(\vec{x}_{i}^{*}\right) \leq \vec{\tau}
$$

Optimizing value as shown above is one possible way to combine multiple objectives (e.g., revenues, market share, brand image, technical performance, etc.) into a single overall profit function.

\section{Multi-Objective Form}

When multiple objectives or product attributes cannot be easily transformed into a single performance metric, a different approach is needed. An approach is proposed similar to that of value analysis (Miles 1972), in that the different performance attributes of a product are considered separately and are then compared with the investments required to obtain such a performance.

In this case, for each product $i$ a performance vector, $\vec{y}$, is optimized consisting of $j$ performance attributes, $y_{j}$, instead of a single expression for value. Generally, maximizing any one of the performance metrics results in different solutions, and so the design team must solve this as a set of metrics to interactively trade-off to determine a solution. As in value analysis, this is formulated against the investments required to achieve any of the performance values. General$l y$, the idea is to find

$$
\vec{x}_{i}^{*}: \vec{y}\left(\vec{x}_{i}^{*}\right)=\max _{\vec{x}_{i}} \frac{\vec{y}}{\sum \text { Investments }}
$$

subject to meeting the same set of constraints as in equation (2).

The general statement in equation (3) is expanded to apply specifically to the development of platform-based product families. The case where a firm needs to select a platform design to develop is considered, that is, whether or not to invest in a particular set of technology to use as a platform in many product variants.

There are two types of investments that need to be made: an initial investment, $I_{0}$, to begin development of the platform technology, and additional investments, $I_{i}$, to derive product variants based on the developed platform. The initial investment may be for R\&D costs such as prototype phases, technology development, or common elements design. The subsequent investments are all those required to launch the variants derived from the platform, such as manufacturing and setup costs, marketing, etc. Once a variant $i$ has been developed, it will be launched and the resulting outcomes, $\vec{y}$ s, will be realized by the organization.

Both the investments and outcomes described above are, generally speaking, a function of the design choices that are made for the product family, as well as a function of uncertainty present during the development process, $\vec{u}$. For example, the investments needed to create variants based on a particular platform depend on the chosen design for that platform; an inflexible platform may require many costly adjustments to create a finished product, while a flexible choice would make it less expensive. Also, choosing a known technology for the platform would increase the probability of ending up with functioning variants. However, at the same time, a new technology may increase the outcomes that can be realized by offering novel variants into the market.

To model this, consider the outcome for a platform as the outcomes of the whole family of products. Over the uncertainty, consider the expected individual product performances:

$$
E\left[y_{i}\right]=\int_{U} y_{j} p d f(\vec{u}) d \vec{u}
$$

Furthermore, the design variables, $\vec{x}_{i}$, that describe each product $i$ are split into the following two groups: $\vec{x}_{p}$, which describes the design of the platform, and $\vec{x}_{V_{i}}$, which describes the design of 
the unique portions of each variant. For the case described above, equation (3) then becomes

Find

$$
\begin{aligned}
V\left(\vec{x}_{p}^{*}, \vec{x}_{v_{i}}^{*}\right) & =\max _{\vec{x}_{p}, \vec{x}_{v_{i}}} \frac{E[\vec{y}]}{E\left[I_{0}+\sum_{i} I_{i}\right]} \\
& =\max _{\vec{x}_{p}, \vec{x}_{v_{i}}} \frac{\int_{U} \vec{y} p d f(\vec{u}) d \vec{u}}{\int_{U}\left(I_{0}+\sum_{i} I_{i}\right) p d f(\vec{u}) d \vec{u}}
\end{aligned}
$$

s.t.

$$
\begin{aligned}
& \operatorname{Pr}_{U}\left(\vec{g}_{i}\left(\vec{x}_{p}, \vec{x}_{v_{i}}, \vec{u}\right) \leq \vec{\tau}_{i}\right) \geq \text { Confidence factor } \\
& \operatorname{Pr}_{U}\left(I_{0}+\sum_{i} I_{i} \leq B\right) \geq \text { Confidence factor }
\end{aligned}
$$

where $\vec{x}_{p}$ includes the design variables describing the platform, $\vec{x}_{v i}$ includes the design variables describing individual portion of variant $i,\left(\vec{x}_{p}^{*}, \vec{x}_{v_{i}}^{*}\right)$ includes the optimum family design (both platform and variants), $\vec{y}$ includes the expected outcome or performance attribute for the product family, $I_{0}$ is the original investment to create the platform, $I_{\mathrm{i}}$ is the investment to commercialize product $i$, factor in discounts, $\vec{x}_{i}$ is the vector of constraints for product $i, \vec{u}$ is the uncertain factors during the development of the product family, $U$ is the uncertainty space for development of the family, $\vec{\tau}_{i}$ is the requirements for performance of product $i$, and $B$ is the budget for the product family.

In other words, both the platform and variant designs are found that maximize the value to the firm, in the spirit of value analysis. This design for the platform must meet the performance targets for each of the supported variants within a given confidence factor. The performance of each variant is given by the platform design choices, the variant design choices, and uncertain factors $\vec{u}$ (e.g., variation in the final performance of the platform). Also, the total investments must fall within the family budget constraints. Again, these investments depend on the platform and variant design choices, and un- certain factors (e.g., variation in the final cost of the platform). These investments may include discounts for sharing a common platform as derived elsewhere (Gonzalez-Zugasti, Otto, and Baker 1999). Finally, both the probabilities of creating a variant as well as the outcomes of each variant are also a function of the chosen platform and variant designs, the targets for the variant, $\vec{\tau}_{i}$, and uncertain factors (e.g., variation in the market acceptance of a variant).

\section{Hpproach}

In this section a practical method to achieve the optimization statement of equation (5) is presented. While for small designs equation (5) can be solved through a traditional search algorithm, for large, complex designs this is not possible for several reasons. First, the knowledge to perform that kind of analysis usually resides in different experts and departments within any real organization. Obtaining the necessary information from all these sources (such as technical and marketing experts) and condensing it into a whole model that can be the basis for a numerical model is infeasible, both logistically, and because many of the constraint limits just cannot be stated a priori. Usually, very large products are designed by concurrent teams of experts who trade off different design parameters, come to understand constraint limits, and interactively negotiate a feasible solution.

A second reason that makes this type of problem very complicated is the fact that evaluation of the performance $\vec{y}$ for large systems is time consuming and expensive, not just in evaluation times of simulations but in handwork needed to set up each evaluation with proper conditions that vary. This limits the number of possible solutions that can be sufficiently explored. When many uncertain factors exist about the development of the products, this complexity problem is compounded.

The complexity of solving the problem leads us to an approach of splitting it into phases: first, a technical analysis phase, where the technical aspects of the design can be worked out, and then an evaluation phase, where the designs can be 
compared, including uncertainties associated with developing the product family.

The overall approach consists of five main steps. The first step is the technical design phase, in which technically feasible designs are created for each alternative product family to be considered. The remaining steps form the evaluation and selection phase. In the second step, a model of the development of each of the identified technically feasible designs is generated. Then in the third step, the uncertain inputs to the development models are assigned based on the current knowledge of these factors. The fourth step is to calculate each of the values used to compare the designs. Finally, in the fifth step these values are used to qualitatively compare the competing designs and select one or explore new platform concepts. Each of these steps is described in detail in the following sections.

\section{FIRST STEP: ALTERNATIVE PRODUCT FAMILY DESIGNS}

In this step, technically feasible designs for a platform-based family of products are created when uncertainties are fixed. That is, the uncertainty in results of the development process is fixed at expected input values, and the design analysis conducted. The result of this step is a finite set of design solutions and their technical performance ratings. There are multiple design solutions over the Pareto frontier of the multiple evaluation criteria $\vec{y}$ involved. Different stakeholders in the design process may have differing weightings of the objectives, and so several designs may be considered to be reasonable solutions, pending a subsequent uncertainty analysis.

The technical analysis phase may be achieved through different means. For very complex designs, this would likely involve a concurrent design team with representatives from all disciplines involved. For simpler platforms or for rough studies of larger systems, this step might be performed with a product model.

However, regardless of the means used to determine the platform design candidates, the basic structure behind this step is the following. First, the platform alternative must be chosen. In other words, the design variables that describe what will be common to all the products in the family need to be chosen. This can be different for different platforms, all using the same technology, or may consist of entirely different technologies with entirely different system equations. Once a candidate platform is specified, the remainder of each product variant's design is completed by determining the variant variables $\vec{x}_{v i}$. These variables would be chosen to optimize the performance of the product variant, subject to meeting the constraints imposed by the specifications of each variant. Once the platform and variants have been determined, the resulting performance ratings for the platform can be determined. If the resulting performance is not adequate, then design iteration would be required among the variant and platform determination. For example, the choice of platform may be changed, and the variants would be adjusted to the new platform design, and a new performance calculated. This would be repeated until adequate solutions were found.

In this technical analysis phase, all uncertain development factors $\vec{u}$ are held fixed. This assumes that the calculated designs describe the products that will be built during actual development. In reality, the performance requirements of the variants will likely shift with changes in the market, technology improvements, etc., and are therefore uncertain at this earlier time when the platform is selected for development. Therefore, the platform evaluation and selection method need to account for that uncertainty. In the following steps, the technically feasible designs created in this first phase will be compared by reintroducing the uncertainty $\vec{u}$.

\section{SECOND STEP: PRODUCT FAMILY DEVELOPMENT MODEL}

Real-options concepts are applied to model the development of each platform-based family. A real-options approach allows for decisions to be made over time, with uncertainty being resolved between decision points. This approach thereby 


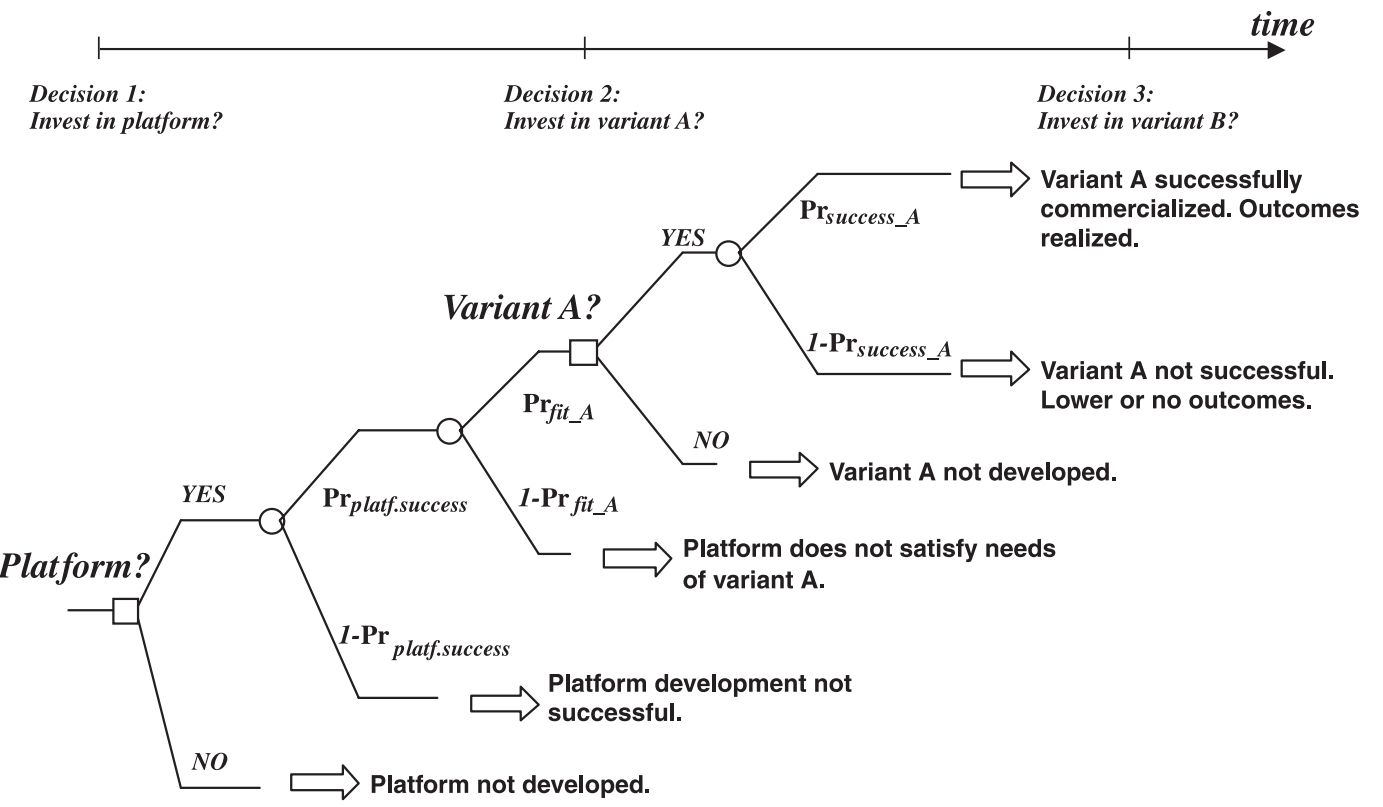

Figure 1: Generalized Platform-Based Product Family Development

includes the value of design flexibility, or the ability to make decisions after uncertain development steps are resolved, such as knowing the outcome of a prototyping stage.

Consider the case where an organization needs to make a decision whether or not to fund a particular platform development, which will in time develop into a family of products. Therefore, the downstream launch decisions must be modeled for each product variant, for each considered platform alternative. The entire set of decisions in the total family platform design/development process is represented as a decision tree with uncertainty and decision nodes. The tree is evaluated by rolling performances back from the multiple possible leaf-node outcomes through the decision and uncertainty nodes to arrive at the trunk-node decision of whether to invest in the particular platform alternative represented by the decision tree.

As shown in Figure 1, the development process begins with a decision (square node) at the root of the tree. If the platform is deemed worthwhile, development begins; otherwise, this particular platform alternative is abandoned. The funding decision is usually followed by test stages, when technologies are verified, or prototypes built to prove the performance of the platform. These are represented by uncertain nodes (circles), because their outcomes are not determined, but can be associated with probability estimates. Once a platform has been tested and found to be a good fit for a desired variant, the organization needs to make an additional funding decision to determine whether a variant should be developed or not. If the variant is funded, its outcome is still uncertain, so it can be represented by additional uncertain nodes. The tree shown in Figure 1 can be easily restructured to represent different situations, such as launching multiple variants at once, or having more testing stages with more possible outcomes. These and other scenarios can be modeled by different combinations or chance (circular), decision (square), and outcome (triangular) nodes.

\section{THIRD STEP: ASSIGN PROBABILITIES AND OTHER UNCERTAIN INPUTS}

Once the structure of the design/development process has been represented, the uncertainty present in the development process must be assigned probabilities. In Figure 1, three types of probability estimates are shown, representing the uncertainty of three distinct 
design/development phases. The first, $\operatorname{Pr}_{\text {platf.success, }}$ is an estimate of the probability of obtaining a successful platform. The next, $\operatorname{Pr}_{\mathrm{fit}_{i}}$, is an estimate of the probability that the developed platform fits the needs of product $i$, so that it can be developed as a variant based on the platform. Finally, $\mathrm{Pr}_{\text {success }}$ is an estimate of the probability of success of variant $i$ once it is developed and launched; in other words, this is the probability of obtaining a particular outcome from variant $i$. These probabilities are generally functions of the chosen platform and variant designs, $\vec{x}_{p}$ and $\vec{x}_{v_{i}}$, the target and outcome performance for the desired products, $\vec{\tau}_{i}$ and $\vec{y}_{i}$, and the uncertain factors, $\vec{u}$. These uncertain factors may be internal (related to the designs), such as the cost of a test stage, or external (unrelated to the chosen designs), such as the state of the economy. Then in general terms the probabilities can be expressed as follows:

$$
\begin{aligned}
\operatorname{Pr}_{\text {platf.success }} & =f\left(\vec{x}_{p}, \vec{u}\right) \\
\operatorname{Pr}_{\text {fit }_{i}} & =f\left(\vec{y}_{v_{i}}, \vec{\tau}_{i}, \vec{u}\right)=f\left(\vec{x}_{p}, \vec{x}_{v_{i}}, \vec{\tau}_{i}, \vec{u}\right) \\
\operatorname{Pr}_{\text {success }_{i}} & =f\left(\vec{x}_{p}, \vec{x}_{v_{i}}, \vec{u}\right)
\end{aligned}
$$

In other words, the probability of developing a successful platform is a function of the design variables that describe the platform. The probability that a product $i$ can be built as a variant based on the given platform is given by the target performance and the performance of the product built as a variant, which in turn is given by the choice of platform and variant design variables. The probability of success of a variant is also given by the design variables that describe the platform and variant design. Finally, all three probabilities are subject to uncertain factors, $\vec{u}$.

This formulation is one that can be used early in the design process to see the effect of changing platform variables on the outcomes of the product family. It is also in a form that makes it possible to obtain the necessary inputs (e.g., the probability estimates) from different experts within the organization. Technical, manufacturing, and marketing experts (among others) can contribute to assign values or distributions to the uncertain inputs of the model.

\section{FOURTH STEP: ROLL BACK TO OBTAIN VALUES}

Once the development trees are complete for each possible design, the value of the platform alternative can be calculated. If the outcomes of all the variants were expressed in monetary terms alone, these outcomes could be rolled back to the base of the tree using standard realoptions calculations to obtain a single measure of value: the expected value of revenues minus costs over the uncertainty. However, with multiple objectives, the tree models need to be calculated in a different manner. The multi-objective approach in equation (5) can be implemented as follows.

Starting with the outcome leaf-nodes of the tree, the expected value of each performance metric is calculated at each of the chance nodes in the tree by multiplying the outcomes of each branch by its associated probability and summing all branches. This is similar to the chance nodes in a single-metric or standard decision tree, except that now the expected value is calculated for each performance metric separately. Thus, the chance nodes in a multi-objective real-options analysis remain essentially the same.

For decision nodes, however, the formulation becomes different. When dealing with monetary outcomes only, it is sufficient to compare the expected outcome of choosing to fund that stage with the required investment. If the outcome is larger than the investment, the value of the root branch is the difference. If the outcome is lower than the investment, the value at that node is simply zero because the investment is not expected to pay off, so that design and development would not be pursued. When modeling multiple performances, however, the decision needs to be based on all the attributes under consideration. The calculation at each decision node must examine the expected performances attained, and as a vector compare this against investments required to determine if the design and development should be pursued. 


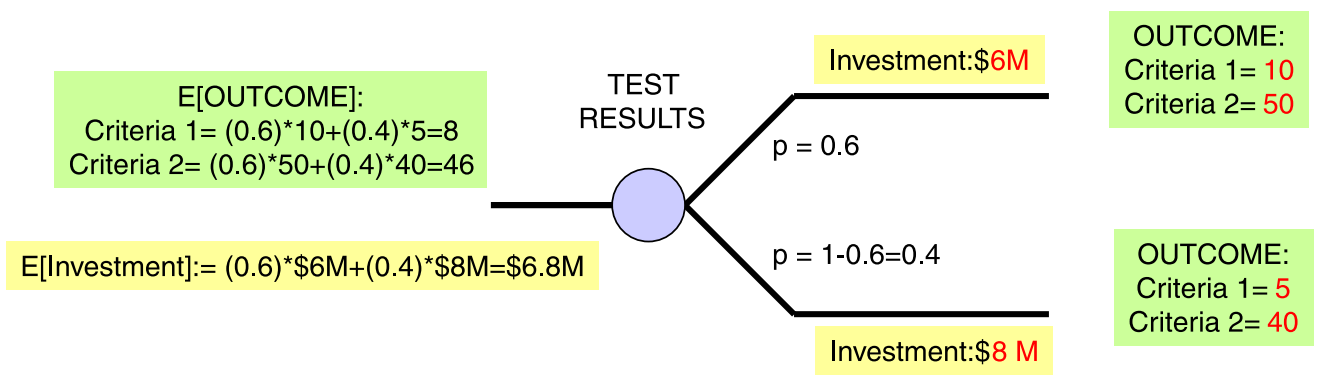

Figure 2: Calculation for Chance and Decision Nodes for Multiple Objectives

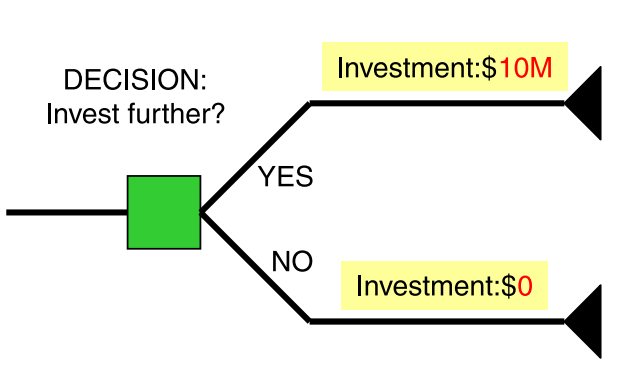

OUTCOME:

Criteria $1=10$

Criteria $2=50$

OUTCOME:

Criteria $1=0$

Criteria $2=0$

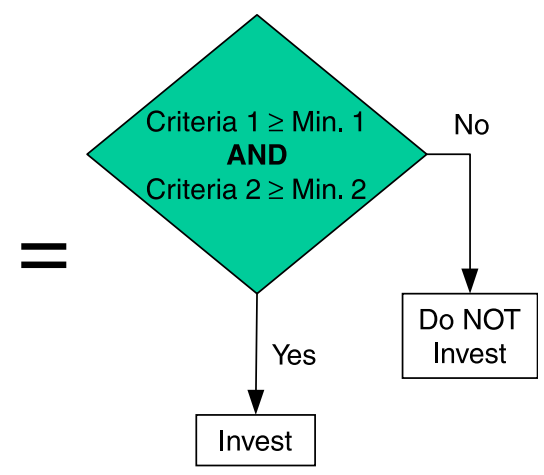

Ideally, each such decision node would reflect the manner in which the decision will in fact be made. There are many models for making such design/development decisions. Elastic weighting or utility functions over the investment and performance outcome space could be constructed, and if sufficient utility is attained, a positive decision is made. Alternatively, fuzzy set imprecision models could be constructed in a similar manner.

In the cases explored, the decisions were made based on meeting minimum performance specifications. A positive decision for design and development is made based on meeting the minimum requirements for each of the performance attributes. Therefore, the decision nodes are modeled in the following way. If all the outcomes are greater than or equal to the minimum performance targets, then the outcomes would be those performance values. On the other hand, if one or more of the outcomes did not meet the requirements, the design would be considered a failure and abandoned, so in this case, all outcomes are set to zero. Figure $\mathbf{2}$ shows a simple example of how a chance and a decision node are calculated for the multiple attribute case.

\section{FIFTH STEP: MULTI-OBJECTIVE SELECTION CHART}

Once the expected outcomes from the development of each platform alternative have been calculated, they can be used to compare the different alternatives and select the most appropriate one. An extended Pugh chart is used to perform this comparative selection. The basis of this method is a qualitative comparison of the alternative platforms based on multiple attributes for the multiple supported products, intended to point out the superior and inferior qualities of each platform. With this, a collective decision can be made.

The first step is to construct a matrix with the alternative platforms as its columns and the performance attributes used for selection as its rows. One of the platform alternatives is chosen by all the stakeholders in the decision as a baseline. Then the remaining alternatives are compared with the baseline on each attribute 
independently. A platform alternative that is superior to the baseline is assigned a " + ," a platform inferior to the baseline is assigned a “,- , and a similar performing platform is given an "S." This process continues until all elements of the matrix have been rated. Each column is then totaled, and superior ranked platforms would exhibit higher positive scores. The main point of the exercise, however, is not to select the highest-scoring platform but to identify the strong and weak areas of each and to try to generate alternative possibilities that combine the good attributes of the original choices and reduce the weaknesses.

\section{Application}

In this section, the approach and implementation models are applied to the design and evaluation of a set of high-speed ships to be built for the Navy for use in a variety of missions. These ships could be built based on a number of different hull shapes. Some of these hulls are proven designs, with fairly well-known performance characteristics and costs, whereas others are more radical designs that promise higher performance, but carry more uncertainty both in costs and in ultimate performance specifications. The purpose of the analysis shown in this paper is to help select one of these hull designs, considering multiple performance attributes and the required investments. This section shows how the approach shown in the previous section can be applied to this example.

\section{CURRENT METHODS OF PRODUCT PLANNING IN SHIP DESIGN}

Current methods for concept design exploration vary widely from organization to organization. In the Naval Construction and Engineering Program at MIT, the methods for performing ship concept design exploration and trade-off analysis consist of

selecting the salient measures of performance (MOP) to use for defining ship performance; determining the ship MOP using synthesis tools; translating the MOP into warfighting measures of effectiveness (MOE) through a mathematical formulation;

combining the MOE into an overall measure of effectiveness (OMOE) through a mathematical formulation;

- estimating cost with or without uncertainty methods;

comparing alternative variants OMOE versus cost, searching for, and selecting Pareto optimal designs; and

applying a rule, such as cost-as-an-independent-variable (CAIV), to select a best variant from among the Pareto set of alternatives.

The MOP, such as speed, stability, seakeeping indices, etc., can be found from synthesis tools such as a spreadsheet tool created by the Maritime Applied Physics Corporation (MAPC), the MIT Math Model, NavCad, or ASSET. These tools use various methods to estimate predicted ship performance, and all have varying levels of design accuracy and different levels of appropriateness for the design phase. This study uses a modified MAPC model for the ship synthesis. This tool uses regression techniques to model the resistance, powering, weights, and other fundamental characteristics. Because the point of the current study is not so much to provide a realistic design result but rather to demonstrate the real-options decision-making methodology, a tool that provided assessments of non-conventional designs was needed. The MAPC tool allows rapid investigation of a range of ship types beyond conventional displacement monohulls, and this provides the necessary real-options problem characteristics of design outcome uncertainty.

The MOP are then translated to MOE and combined to form an OMOE using a hierarchical weighted sum method using a spreadsheet (Whitcomb 1998). Cost was determined through the MAPC tool using a modification to the original cost method to account for probable cost increases due to militarization of the commercial ships in the MAPC database. 
The limitation of the trade-off method described above is that it is difficult to account for cost uncertainty and risk in the down select process. Cost methods, such as treating cost as a random variable and using Monte Carlo simulations, can account for component cost uncertainty and the relationship to the overall ship cost (Garvey 1999). When used in conjunction with the OMOE models, these methods can account for an overall cost risk, but they cannot explicitly account for the uncertainty in obtaining ranges of effectiveness. The methods especially do not account for decision-making possibilities during the ship product development phase. The real-options method allows the decision maker to see the span of possibilities involving ranges of cost and effectiveness outcomes over the project development period. The method applies particularly well to products that offer the possibility of superior effectiveness, but at a high risk of obtaining the desired effectiveness.

\section{HIGH-SPEED SHIP EXAMPLE}

The hull alternatives considered in this example include a catamaran, a surface-effect-ship (SES), and a hydrofoil small waterplane area ship (HYSWAS) design. Each of these platform alternatives has its conceptual technical strengths and weaknesses. For example, the catamaran is a well-known design, currently used for ferrying passengers and automobiles in a relatively stable, high-speed platform. The Australian catamaran HMAS Jervis Bay recently showed high-speed littoral support possibilities in the Indonesian theater. The SES hull forms have been built and used for high-speed applications; however, skirt designs remain challenging for larger ships, higher sea state operation, and higher speeds. The HYSWAS is an experimental ship combining the advantages of hydrostatic lift of a displacement hull with the dynamic lift provided by hydrodynamic lifting bodies. The three options have different investment requirements, risk levels, and expected performance, yet provide intriguing possibilities for use in high-speed military platforms for littoral ocean area applications.
In this case, the platform is a common hull design that would be the base for each of the desired ships. Possible platforms range from standard high-speed ship designs such as hydrofoils to radically new designs such as HYSWAS hulls. The variants are the finished ships that are built based on the chosen platform. In this example, the three desired variants are a medical (MED) high-speed ship, a surface-fire-support (SFS) high-speed ship, and a logistics support (LS) high-speed ship. Each of these variants will also be used in different scenarios, in this case, the Mediterranean Sea, Atlantic, Indian, and Pacific Ocean scenarios. Finally, the fleet of ships that share a common hull platform as the product family is considered.

The situation is explored where a program to develop a fleet of these high-speed ships has just begun. The main question to be answered in this case is which platform should receive the required initial funding and begin development.

\section{APPLICATION EXAMPLE}

In the Approach section, five main steps involved in applying the proposed method to a product family design problem are described. Each of the steps is applied to the high-speed ship example below.

Step 1-Product Family Designs - The alternative product families or sets of desired ships were created through the use of a product model that allowed the specification of design variables and returned a set of performance attributes for the given ship. The model used in this example was a modified version of the MAPC spreadsheet for evaluation of high-speed ship designs based on various hull types.

Once the product model exists, the next step is to specify the platforms to be explored. For this example, three hull types were chosen:

Catamaran, SES, and HYSWAS. Then, each mission or variant was specified. Table 1 shows the minimum required specifications for each variant, as well as the ideal target for each performance attribute. The ideal values for speed, 


\begin{tabular}{|c|c|c|c|c|}
\hline Criteria & Variant & Minimum & Ideal & Units \\
\hline \multirow[t]{3}{*}{ Speed in waves } & MED & & & \\
\hline & SFS & 35 & 40 & Knots \\
\hline & LS & & & \\
\hline \multirow[t]{3}{*}{ Payload } & MED & 150 & 300 & \\
\hline & SFS & 150 & 400 & Long tons \\
\hline & LS & 150 & 500 & \\
\hline \multirow[t]{3}{*}{ Range } & MED & 1,500 & 2,000 & \\
\hline & SFS & 1,500 & 2,500 & Nautical miles \\
\hline & LS & 1,500 & 3,000 & \\
\hline
\end{tabular}

payload, and range for each desired variant were input to the performance model. The model was then run to find the actual performance of each variant based on each of the hull platforms and for each of the given scenarios shown in Table 2. The result was a set of performance ratings for each combination of scenario, mission, and hull.

The performance attributes returned by the model were the expected speed of the ship in waves, payload, range, and cost. Each set of these performance attributes is the outcome of developing a given variant based on a particular hull and used in a given scenario. These individual outcomes can then be totaled to find the expected performance for the whole fleet.

However, the performance outcomes do not always meet the minimum requirements for a variant. For example, the expected sea state for a given scenario may affect the range or payload of a mission enough to cause it to fail the minimum required performance under that scenario. Then, following the logic shown in Figure 2 for making funding decisions, this particular ship would not be built for that scenario. No additional investment would then be made, and the performance outcomes for that scenario and mission would equal zero. The fraction of the desired fleet that is successful, or meets all minimum requirements, can be calculated as follows:

$$
f_{s}=\frac{\sum_{k} \sum_{l}\left(r_{\mathrm{min}} \cdot n_{\mathrm{req}}\right)_{k, l}}{\sum_{k} \sum_{l}\left(n_{\mathrm{req}}\right)_{k, l}}
$$

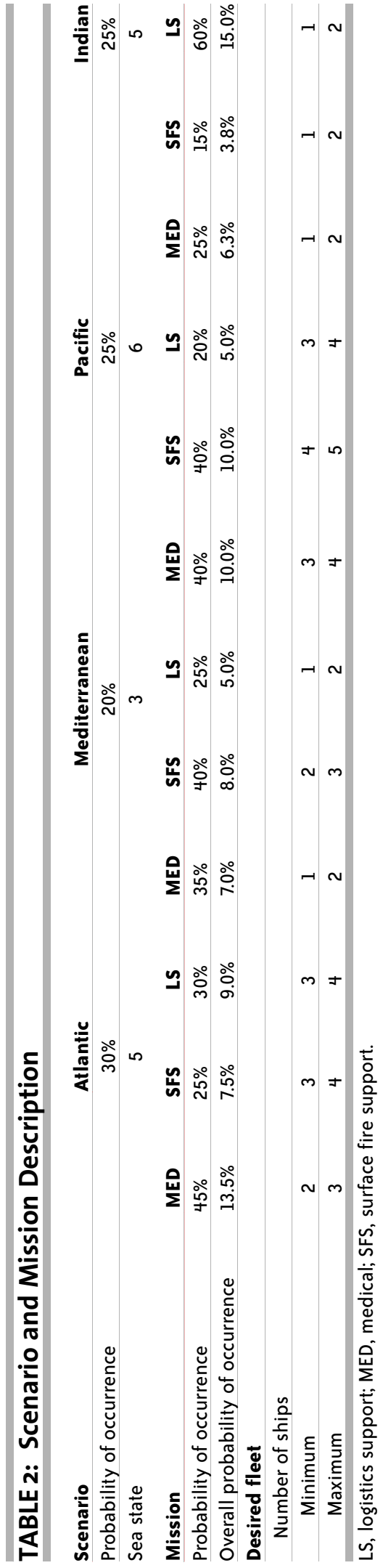

NAVAL ENGINEERS JOURNAL 
where $f_{s}$ is the successful fraction of fleet.

$r_{\min }= \begin{cases}1 & \text { if all minimum requirements are met } \\ 0 & \text { otherwise }\end{cases}$

where $n_{r e q}$ is the number of ships required, $k$ is the subscript denoting type of mission (MED, SFS, LS), and $l$ is the subscript denoting scenario (Atlantic, Pacific, Indian, Mediterranean).

In addition, the expected fleet performance must be weighed by both the probability of occurrence of a given mission and scenario, and by the number of ships that are required by each of those combinations. The performance ratings for a mission with a high probability of occurrence are then weighed more heavily than one that is not expected to happen as often. Similarly, performances for a mission that requires more ships are also weighed more heavily than one that requires few ships. The result is then a weighed fleet performance rating for speed, range, and payload. Equation (8) shows how the performance attributes were totaled to obtain the weighed expected fleet performance:

$$
\bar{y}=\frac{\sum_{k} \sum_{l}\left(y \cdot \operatorname{Pr}_{\mathrm{occ}} \cdot r_{\mathrm{min}} \cdot n_{\mathrm{req}}\right)_{k, l}}{\sum_{k} \sum_{l}\left(\operatorname{Pr}_{\mathrm{occ}} \cdot n_{\mathrm{req}}\right)_{k, l}}
$$

where $\bar{y}$ is the weighted fleet performance, and $\mathrm{Pr}_{\text {occ }}$ is the probability of occurrence of mission $k$ in scenario $l$.

The fraction of the fleet that is successful can be similarly weighed as follows:

$$
\bar{f}_{s}=\frac{\sum_{k} \sum_{l}\left(\operatorname{Pr}_{\mathrm{occ}} \cdot r_{\mathrm{min}} \cdot n_{\mathrm{req}}\right)_{k, l}}{\sum_{k} \sum_{l}\left(\operatorname{Pr}_{\mathrm{occ}} \cdot n_{\mathrm{req}}\right)_{k, l}}
$$

Finally, the total fleet cost is also calculated by aggregating the cost of all missions that meet the minimum requirements and are therefore considered successful and funded.

Step 2-Product Family Development ModelsAfter the desired products have been modeled and the outcomes of each family have been found, the development path for each family must be modeled. In order to do that, a decision tree is created to represent the stages of development of each alternative family. In this case, three trees were needed to model the Catamaran-, SES-, and HYSWAS-based alternative fleets. Figure 3 shows one of those trees.

At the root of each of the trees is the current decision that needs to be made: whether or not to make the initial investment into the platform (or hull) development. If the project is funded, there is an uncertain event node representing the uncertainty in the development of the hull, with two possible outcomes: a high-performing hull or an average-performance hull. Each of these branches then meets another uncertain node representing the uncertainty in fit between the capabilities of the developed hull and the current needs of the desired fleet. Again, these second nodes are modeled as having two possible branches: good fit or poor fit between hull and mission requirements. Each of these four branches then leads to a second decision node: whether or not to fund the fleet of ships based on the given platform hull. If this second funding round is made, the outcomes of building the fleet are realized; otherwise, the outcomes (and additional investments) are zero because the fleet is not built. These outcomes are nominally the performance outcomes found in Step 1. However, these performance attributes are likely higher if the development goes well at every step or node, and likely lower if the development follows a less successful path. Therefore, the nominal performance ratings are modified by a performance multiplier that varies according to the development path. The most successful path then will produce higher than nominal performance ratings, while the less successful paths will produce lower than nominal ratings. Alternatively, development path could be modeled as always producing the same performance outcome, but with different paths requiring different levels of investment or effort. This latter approach may apply better to other types of products. However, the former method more 
Figure 3: Development Model for Highspeed Ship Fleet

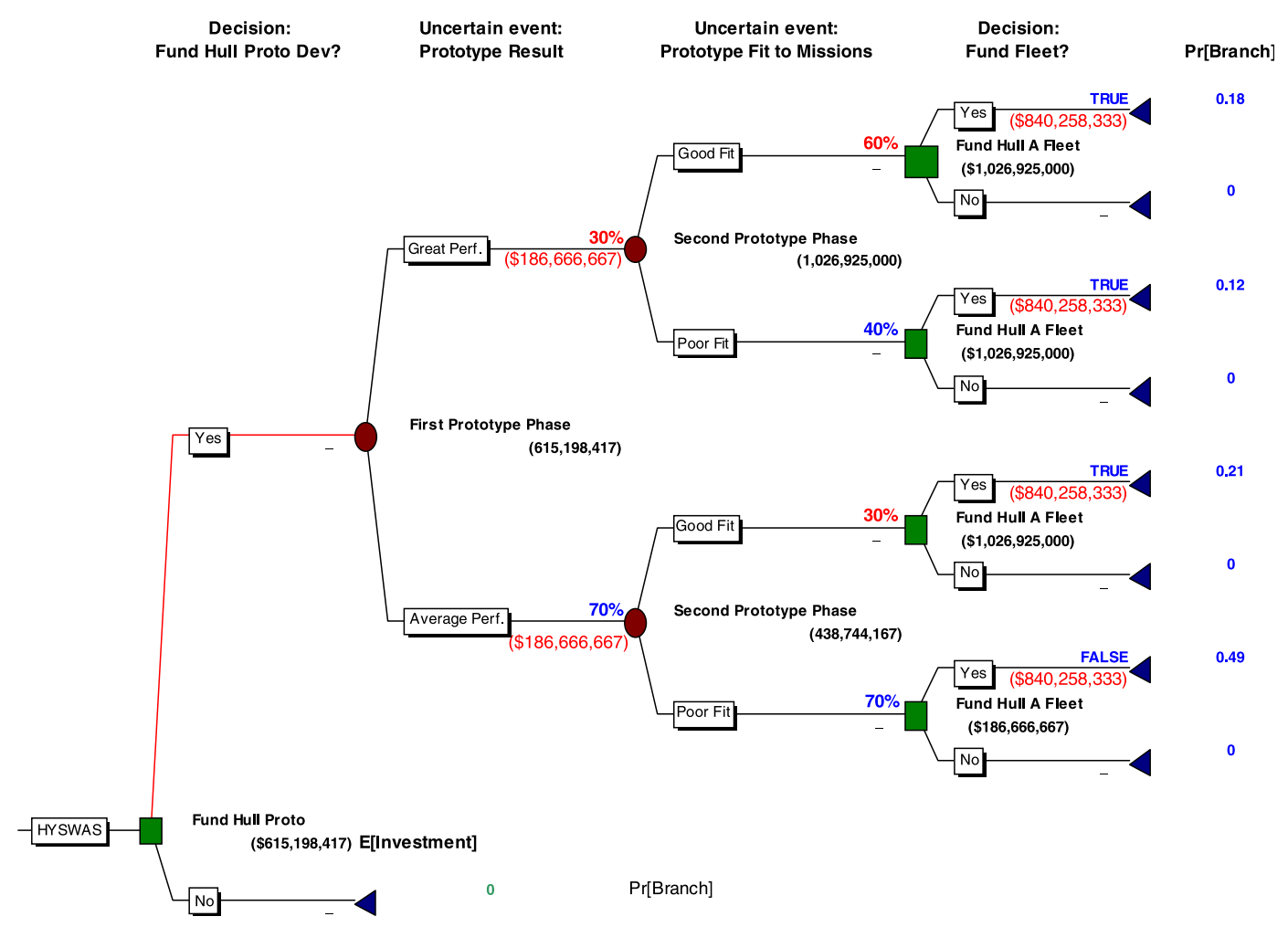

closely resembles the development of naval products, because usually projects have a fixed budget, and project scope (and thus the resulting performance) is adjusted to fit within those budgetary constraints.

Step 3-Assign Probabilities and Other Uncertain Inputs-Once the three family (or fleet) development trees were built, all their inputs had to be entered before their value could be calculated. Inputs that were well known were represented as fixed values. The remaining, uncertain inputs were represented by probability density functions (pdfs). For this example, reasonable values and uncertainty ranges were chosen based on data from past ship development projects. In different situations, these inputs may be outputs of cost models or expert assessments when those data are available. Table 3 shows the types of inputs that were entered into the trees, and their associated uncertainty.

Step 4-Roll Back to Obtain Values-Once all inputs to the development models have been de- fined, the value of each family can be calculated. This is carried out by rolling back the outcomes at the end of the trees through the chance and decision nodes as shown in Figure 2 until the base of the tree is reached.

Monte Carlo simulation software can be used to model the distributions in uncertain inputs and to calculate the distribution of the outputs of the model output, or the expected values for performances and investments for each tree or fleet. Different scenarios can be explored by changing the inputs and repeating the simulation. For example, it is possible to calculate the values of each of the platforms for cases where the required number of ships is larger or smaller than the nominal.

The figures below summarize the results of the analysis for one such scenario of high-speed ship designs. Figure 4 shows the expected performance/investment results for all three platformbased fleets. The SES-based fleet exhibits the highest performance per invested dollar, 


\section{TABLE 3: Development Tree Input Types}

\begin{tabular}{|c|c|c|c|}
\hline Input & Platform & Distribution & Parameters \\
\hline \multirow[t]{3}{*}{ Initial investment } & CAT & & Nominal $=\$ 100,000,000 ; \quad$ Minimum $=-30 \% ;$ Maximum $=+10 \%$ \\
\hline & SES & Triangुular & Nominal $=\$ 150,000,000 ; \quad$ Minimum $=-30 \% ;$ Maximum $=+10 \%$ \\
\hline & HYSWAS & & Nominal $=\$ 200,000,000 ; \quad$ Minimum $=-30 \% ;$ Maximum $=+10 \%$ \\
\hline \multirow[t]{3}{*}{ Fleet investment } & CAT & & \\
\hline & SES & Triangular & Nominal $=$ Successful ships $\times$ Cost/ship.; Minimum $=-10 \% ;$ Maximum $=+5 \%$ \\
\hline & HYSWAS & & \\
\hline \multirow[t]{3}{*}{ Pr(Platform success) } & CAT & & $80 \%$ \\
\hline & SES & Fixed & $80 \%$ \\
\hline & HYSWAS & & $30 \%$ \\
\hline \multirow[t]{3}{*}{$\operatorname{Pr}($ Fit $)$} & CAT & & Branch $1=60 \%$; branch $2=50 \%$ \\
\hline & SES & Fixed & Branch $1=60 \%$; branch $2=50 \%$ \\
\hline & HYSWAS & & Branch $1=60 \%$; branch $2=30 \%$ \\
\hline \multirow[t]{6}{*}{ Performance multipliers } & CAT & & Branch $1=1.1-1.3$; branch $2=0.9-1.0$ \\
\hline & & & Branch $3=0.9-1.1 ;$ branch $4=0.8-1.0$. \\
\hline & SES & Uniform & Branch $1=1.1-1.4 ;$ branch $2=0.9-1.1$ \\
\hline & & & Branch $3=0.9-1.0 ;$ branch $4=0.6-1.0$. \\
\hline & HYSWAS & & Branch $1=1.1-1.7$; branch $2=0.9-1.3$ \\
\hline & & & Branch $3=0.8-1.0 ;$ branch $4=0.3-1.0$. \\
\hline \multirow[t]{3}{*}{ Number of ships required } & CAT & & \\
\hline & SES & Discrete & Nominal, $p=75 \%$. Maximum $=$ Nominal $+1, p=25 \%$ \\
\hline & HYSWAS & & \\
\hline
\end{tabular}

CAT, catamaran; HYSWAS, hydrofoil small waterplane area ship; SES, surface-effect-ship.

followed by the catamaran-based fleet, and lastly by the HYSWAS fleet.

However, Figure 5 shows the successful fraction of the desired fleet for each platform-based family of ships. The SES fleet, even though it exhibits the highest performance/dollar, is able to meet the desired requirements only in a small fraction of missions. At the other extreme, the HYSWAS fleet provides the lowest performance/investment ratings, but is able to meet the mission requirements in most cases. These conflicting results in various criteria lead us to the next step in the approach, where the fleets are compared qualitatively over the multiple dimensions that need to be considered.

Step 5-Multi-Objective Selection Chart-Based on the results obtained in Step 4, a design team can begin to compare the different alternatives. This can be done by constructing a Pugh chart as shown in Table 4. First, a baseline design is chosen; in this case, the chosen baseline is the catamaran-based fleet. Then the HYSWAS- and
SES-based fleets are rated compared with the baseline along the measures of value defined in the Approach section of this paper, as well as the initial investments required and the fraction of the fleet that meets the desired mission requirements.

In this example, the SES-based fleet shows better performance/investment but lower successful fleet fraction than the baseline catamaran fleet. Even though the numerical total at the bottom of the SES column is higher than the baseline, it is not very large, and the relative importance of the different comparison criteria and the list of criteria used must be considered. For instance, if the first three criteria were condensed into a single one such as Expected Performance/ Investment, the numerical score would be different. The main purpose of this qualitative comparison is to provide a method for the design team to come to a consensus as far as the importance of different criteria is concerned, what the right list of criteria should be, and to point out other possible designs that may combine the good aspects of the explored platforms. 
Figure 4: Performance/Investment Ratings for Three Platform-Based Fleet Designs
Figure 5: Fleet Fraction that Meets All Mission Requirements.
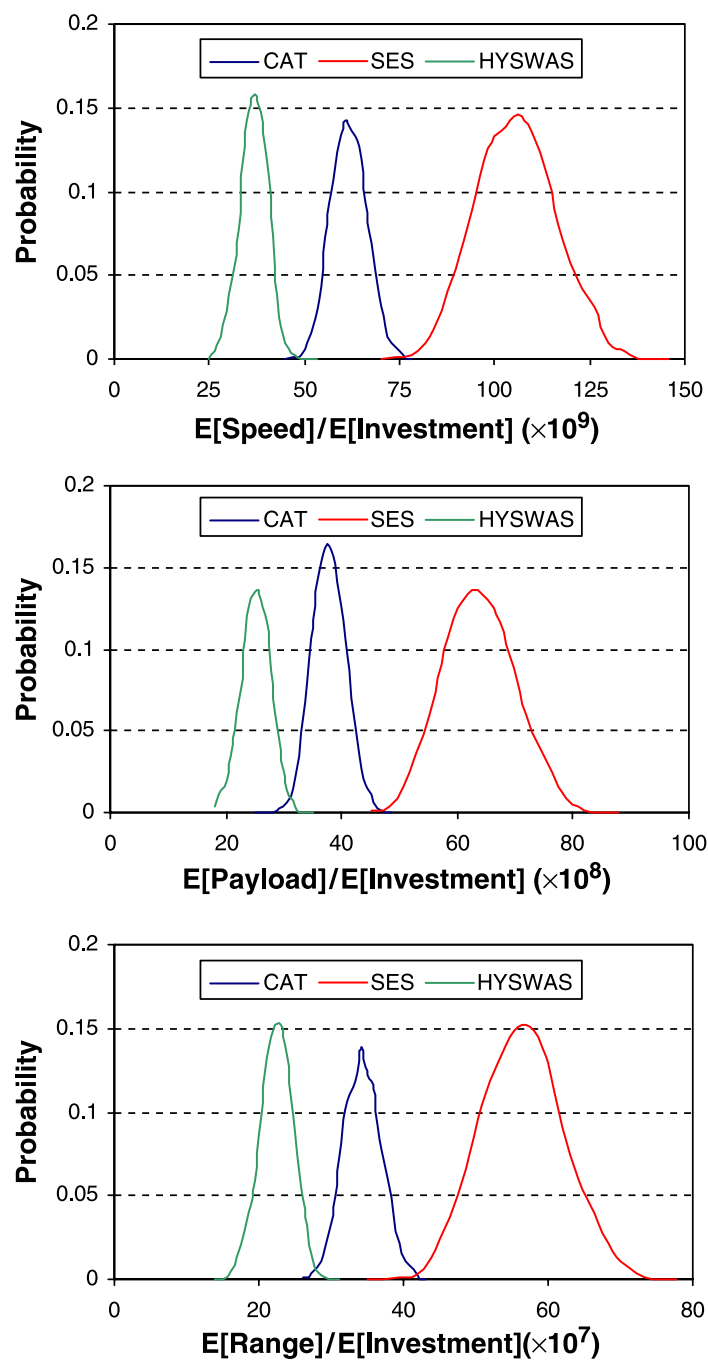

\section{Conclusion}

The real-options formulation has been presented for selecting platforms when considering multiple performance objectives under uncertainty.

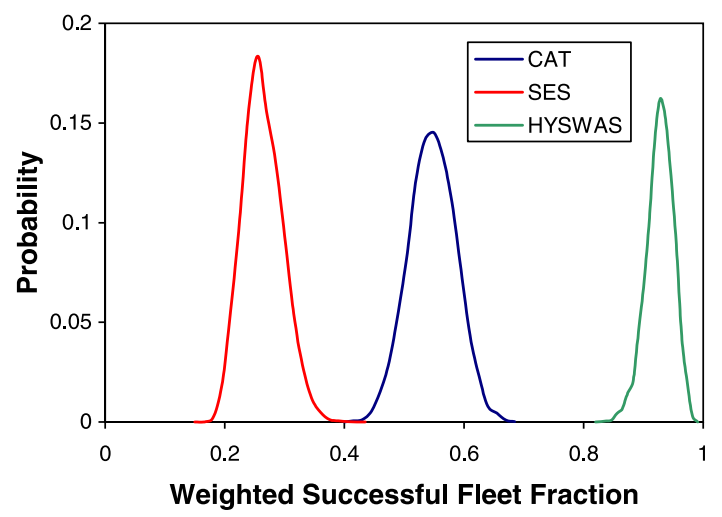

TABLE 4: Qualitative Comparison of Platform-Based Fleets

\begin{tabular}{lccc} 
& \multicolumn{3}{c}{ Platform } \\
\cline { 2 - 4 } Criteria & Catamaran & SES & HYSWAS \\
Speed/investment & & + & - \\
Payload/investment & & + & - \\
\hline Range/investment & & + & - \\
\hline $\begin{array}{l}\text { Weighted fleet fraction } \\
\quad \text { successful }\end{array}$ & Baseline & - & + \\
\hline Initial investment & & & \\
\hline Total & & - & - \\
\hline & & +1 & -3
\end{tabular}

HYSWAS, hydrofoil small waterplane area ship; SES, surface-effect-ship.

This has benefits and difficulties as compared with the standard approach of considering monetary outcome only. It has benefit in that it is often impossible to transform effectiveness outcomes (benefits) into monetary terms, such as in defense or highly technical projects. On the other hand, the result is a multi-objective decision that must be made, comparing several platform alternatives on several criteria. This difficulty is intrinsic to multi-objective evaluation. Multiobjective formulations are useful when monetary conversion is not possible or accurate, such as when long-term profitability is of more concern than quantifiable short-term profitability, or when outcomes cannot be reasonably quantified.

\section{Hcknowledgments}

The research reported in this document was made possible in part by the MIT Center for Innovation in Product Development under NSF Cooperative Agreement Number EEC-9529140 and a grant from the Office of Naval Research, Award \#N00014-99-1-1090. Any opinions, findings, or recommendations are those of the authors and do not necessarily reflect the views of the sponsors.

\section{References}

Allen, J., F. Mistree, and E. Al, "Fuzzy compromise: An effective way to solve hierarchical design problems," Structural Optimization, Vol. 4, pp. 115-120, 1992.

Brealey, R. and S. Myers, Principles of corporate finance, Fourth Edition, McGraw-Hill. New York, 1991. 
Bremner, R., "Cutting edge platforms." Financial Times Automotive World, pp. 30-38, September 1999.

Dahmus, J.B. and K.N. Otto, "Incorporating lifecycle costs into product architecture decisions," ASME Design Enģineering Technical Conferences, DETC2001/ DAC-21110, Pittsburg, PA, ASME, 2001.

Dixit, A. and R. Pindyck, Investment under uncertainty, Princeton University Press. Princeton, NJ, 1994.

Faulkner, T., "Applying 'options thinking' to R\&D valuation," Research-Technology Management, Vol. 39, No. 3, pp. 50-56, 1996.

Fujita, K., H. Sakaguchi, and S. Akagii "Product variety deployment and its optimization under modular architecture and module commonalization," ASME Desiģn Enģineering Technical Conferences, Las Veģas, NV, DFM-8923, 1999.

Garvey, P., Probability methods for cost uncertainty analysis: A systems engineerinǵ perspective, MarcelDecker. New York, 1999.

Gonzalez-Zuģasti, J.P. and K.N. Otto, "Platform-based spacecraft design: A formulation and implementation procedure." IEEE Aerospace Conferences, Biģ Sky, MT, 2000.

Gonzalez-Zugasti, J.P., K. Otto, and J. Baker, "A method for architecting product platforms with an application to the design of interplanetary spacecraft," DETC98/

DAC-5608, Proceedings of the DETC, Atlanta, GA, 1998.

Gonzalez-Zugasti, J.P., K.N. Otto, and J.D. Baker, "Assessing value for product family design and selection,"

ASME Designn Enģineering Technical Conferences, Las Vegas, NV, DAC-8613, 1999.

Krishnan, V., R. Singh, and D. Tirupati, "A model-based approach for planning and developing a family of technology-based projects," Working paper, University of Texas at Austin, Management Department, April 1998.

Martin, M. and K. Ishii, "Desiggn for variety: Development of complexity indices and design charts," ASME Desiģn Enģineering Technical Conferences, DFM-4359, 1997.

Mistree, F., O. Hughes, and B. Bras, "The compromise decision support problem and the adaptive linear programming algorithm," In Structural optimization: Status and Promise, ed. M. Kamat, AIAA. Washington, DC, pp. 247-286, 1993.
Meyer, M.H. and A.P. Lehnerd, The power of product platforms, The Free Press. New York, 1997.

Miles, L., Techniques of value analysis and engineering, McGraw-Hill. New York, 1972.

Neely, J., Improving the valuation of research and development: A composite of real options, decision analysis and benefit valuation frameworks, Massachusetts Institute of Technology. Cambridge, MA, 1998.

Nelson, S., M. Parkinson, and P. Papalambros, "Multicriteria optimization in product platform design," ASME Desiggn Enģineering Technical Conferences, Las Vegas, NV, DAC-8676, 1999.

Sanderson, S., "Cost models for evaluating virtual design strateģies in multicycle product families," Journal of Engineering and Technoloğy Management, Vol. 8, pp. 339-358, 1991.

Sanderson, S. and M. Uzumeri, "Managging product families: The case of the Sony Walkman," Research Policy, Vol. 24, pp. 761-782, 1995.

Simpson, T., J. Maier, and F. Mistree, "A product platform concept exploration method for product family design," ASME Designn Enģineering Technical Conferences, DTM-8761, Las Vegas, NV, 1999.

Stone, R., K. Wood, and R. Crawford, "A heuristic method to identify modules from a functional description of a product," ASME Design Engineering Technical Conferences, Atlanta, GA, DTM-5642, 1998.

Trigeorgis, L. and S. Mason, "Valuing managerial flexibility," Midland Corporate Finance Journal, Vol. 5, No. 1, pp. 14-21, 1987.

Whitcomb, C., "Naval ship design philosophy implementation," Naval Engíneers Journal, 110, No. 1, pp. 4963, May 1998.

Yu, J., J.P. Gonzalez-Zugasti, and K.N. Otto, "Product architecture definition based upon customer demands," Journal of Mechanical Design, Vol. 121, No. 3 , p. 329, 1999.

Zamirowski, E. and K.N. Otto, "Identifying product portfolio architecture modularity using function and variety heuristics," ASME Desiģn Enģineerinğ Technical Conferences, Las Veģas, NV, DTM-8760, 1999.

Zugasti, J.P., "Models for platform-based product family design," Ph.D. dissertation, Mechanical Engineering, MIT, 2000. 


\section{Huthor Biographies}

Dr. Javier P. Gonzalez-Zugasti is a Principal Engineer and Group Leader, Automation Engineering at TransForm Pharmaceuticals in Lexington, MA. While at TransForm, he has participated in the development of several automated laboratory systems for highthroughput experimentation used to conduct pharmaceutical product research and development. Prior to this position, he was a Research Assistant at the MIT Center for innovation in Product Development. His industrial experience also includes a position as mechanical design engineer at Diagnostic Ultrasound Corp. (now Verathon Inc.), a manufacturer of diagnostic medical devices in Bothell, WA.

Dr. Kevin N. Otto is the founder of Robust Systems and Strategy, a consulting and training firm specializing in helping companies bring new innovations into their products. His expertise lies in systems engineering, design for six sigma, modularity, modeling and analysis, roadmaps, market analysis, design verification, process improvement, and robustness and reliability. Kevin served as an Associate Professor of Mechanical Engineering at MIT, where be co-founded the Center for innovation in Product Development. He is the author of Product Design, and many peer-reviewed academic journal articles.
Dr. Clifford A. Whitcomb, CSEP, is an Associate Professor of Systems Engineering at the Naval Postgraduate School, Monterey, CA. He has 30 years experience in leadership roles in teaching, research, and implementation of naval ship system engineering for the US Navy. Previously, he was a Professor and the Northrop Grumman Ship Systems Endowed Chair in Shipbuilding and Engineering in the School of Naval Architecture and Marine Engineering, and Professor of Engineering Management, at the University of New Orleans, New Orleans, Louisiana. He also beld a position as an Eminent Scientist in the area of naval electric power systems the Naval Sea Systems Command, Naval Surface Warfare Center, Philadelphia, PA. Prior to this, he was a senior lecturer and research scientist in the System Design and Management (SDM) program in the Engineering Systems Division (ESD) at MIT, as well as and Associate Professor of Naval Construction and Engineering in the Ocean Engineering Department at MIT. Prior to his career in academia, Dr. Whitcomb was a naval engineering duty officer, with positions as a program officer at the Office of Naval Research (ONR); Ship Design Research Engineer at the Naval Surface Warfare Center, Carderock, Division, Bethesda, MD; and Shipwork Coordinator at the Supervisor of Shipbuilding, Groton, CT. He is a submarine warfare officer, having served aboard USS Scamp (SSN-588). He is a Certified Systems Engineering Professional (CSEP) and a Lean Six Sigma Master Black Belt. 